\title{
New asymptotics for the mean number of zeros of random trigonometric polynomials with strongly dependent Gaussian coefficients
}

\author{
Thibault Pautrel*
}

\begin{abstract}
We consider random trigonometric polynomials of the form

$$
f_{n}(t):=\frac{1}{\sqrt{n}} \sum_{k=1}^{n} a_{k} \cos (k t)+b_{k} \sin (k t),
$$

where $\left(a_{k}\right)_{k \geq 1}$ and $\left(b_{k}\right)_{k \geq 1}$ are two independent stationary Gaussian processes with the same correlation function $\rho: k \mapsto \cos (k \alpha)$, with $\alpha \geq 0$. We show that the asymptotics of the expected number of real zeros differ from the universal one $\frac{2}{\sqrt{3}}$, holding in the case of independent or weakly dependent coefficients. More precisely, for all $\varepsilon>0$, for all $\ell \in(\sqrt{2}, 2]$, there exists $\alpha \geq 0$ and $n \geq 1$ large enough such that

$$
\left|\frac{\mathbb{E}\left[\mathcal{N}\left(f_{n},[0,2 \pi]\right)\right]}{n}-\ell\right| \leq \varepsilon
$$

where $\mathcal{N}\left(f_{n},[0,2 \pi]\right)$ denotes the number of real zeros of the function $f_{n}$ in the interval $[0,2 \pi]$. Therefore, this result provides the first example where the expected number of real zeros does not converge as $n$ goes to infinity by exhibiting a whole range of possible subsequential limits ranging from $\sqrt{2}$ to 2 .
\end{abstract}

Keywords: random trigonometric polynomials; nodal set; strong dependence.

AMS MSC 2010: Primary 26C10, Secondary 30C15; 42A05.

Submitted to ECP on February 25, 2020, final version accepted on April 20, 2020.

\section{Introduction and statement of the results}

\subsection{Real zeros of random trigonometric polynomials}

There is tremendous amount of literature about complex or real zeros of random polynomials and their asymptotics as the degree of the latter goes to infinity. Recently, the universality of these asymptotics has been established in a certain number of models, see e.g. [Kac43, IM68, Far86, Mat10, Muk18, NNV15, DNV18] in the case of algebraic polynomials and [AP15, ADL, Fla17, IKM16, ADP19] in the case of trigonometric polynomials. The notion of universality stands here for the fact that these asymptotics do not depend on the choice of the law of the random entries, and to a certain extent, nor their

*Univ Rennes, CNRS, IRMAR - UMR 6625, F-35000 Rennes, France

E-mail: thibault. pautrel@univ-rennes1.fr 
correlation.

Our model belongs to the large class random trigonometric polynomials of the form

$$
f_{n}(t):=\frac{1}{\sqrt{n}} \sum_{k=1}^{n} a_{k} \cos (k t)+b_{k} \sin (k t), \quad t \in \mathbb{R},
$$

where $\left(a_{k}\right)_{k \geq 1}$ and $\left(b_{k}\right)_{k \geq 1}$ are two independent stationary Gaussian processes with correlation function $\rho: \mathbb{N} \rightarrow \mathbb{R}$, namely $\mathbb{E}\left[a_{k} a_{l}\right]=\mathbb{E}\left[b_{k} b_{l}\right]=: \rho(|k-l|)$ and $\mathbb{E}\left[a_{k} b_{l}\right]=0$ for all $k, l \geq 1$. Thanks to Bochner's theorem, we then know that $\rho$ is given by the Fourier transform of a finite measure $\mu$, called the spectral measure, and supported on the torus $\mathbb{R} / 2 \pi \mathbb{Z}$. The case where $\rho(k)=0$ for all $k \geq 1$ corresponds to independent Gaussian coefficients as first studied by Dunnage in [Dun66]. Later, in [Sam78] and [RS84], the authors considered the two "extreme" cases where $\left.\mathbb{E}\left[a_{i} a_{j}\right]=\rho_{0} \in\right] 0,1[$ and $\mathbb{E}\left[a_{i} a_{j}\right]=\rho_{0}^{|i-j|}$ respectively.

More recently, the authors of [ADP19] considered the case where the spectral measure admits a density satisfying mild hypotheses. In all these cases, it was shown that $\mathcal{N}\left(f_{n},[0,2 \pi]\right)$, the number of real zeros of the random function $f_{n}$ in the interval $[0,2 \pi]$, obeys the same limit

$$
\lim _{n \rightarrow+\infty} \frac{\mathbb{E}\left[\mathcal{N}\left(f_{n},[0,2 \pi]\right)\right]}{n}=\frac{2}{\sqrt{3}} .
$$

This naturally raises the question of the existence of choices of "exotic" random entries such that the asymptotics of the expected number of real zeros do not coincide with the universal one. In fact, considering standard Gaussian coefficients, one way to obtain asymptotics that do not match $2 / \sqrt{3}$ is to consider palindromic entries as in [FL12, Pir19b] or very special pairwise block entries such as in Theorem 2.3 and 2.4 of [Pir19a].

We consider here the natural and purely singular case where the spectral measure is given by $\mu:=\frac{1}{2}\left(\delta_{\alpha}+\delta_{-\alpha}\right) \Longleftrightarrow \rho(k)=\cos (k \alpha)$, for some real $\alpha \geq 0$. If $\alpha \in \pi \mathbb{Q}$, the correlation function is periodic and the corresponding random coefficients of $f_{n}$ are strongly correlated at arbitrary large distance. If $\alpha \notin \pi \mathbb{Q}$, the sequence $(\rho(k))_{k \geq 0}$ is dense in $[-1,1]$ and the correlations between the random coefficients of $f_{n}$ become really intricate. We shall see that the asymptotics of the number of real zeros of $f_{n}$ then heavily depends on the arithmetic nature of $\alpha$ and more precisely on the distance of $n \alpha$ to $\pi \mathbb{Z}$.

\subsection{Statement of our results}

Naturally, since $f_{n}$ is a random trigonometric polynomial of degree $n$, its number of zeros in bounded by $2 n$. In the case where $n \alpha \in \pi \mathbb{Z}$, we show that the expected number of real zeros is maximal in the following sense.

Proposition 1.1. If $\alpha=0$, then for all $n \geq 1$ we have almost surely

$$
\mathcal{N}\left(f_{n},[0,2 \pi]\right)=2 n .
$$

If $\alpha \in \pi \mathbb{Q}$ then

$$
\lim _{n \rightarrow+\infty}\left|\frac{\mathbb{E}\left[\mathcal{N}\left(f_{n},[0,2 \pi]\right)\right]}{n}-2\right| \mathbb{1}_{n \alpha \in \pi \mathbb{Z}}=0 .
$$

The case $n \alpha \notin \pi \mathbb{Z}$ is more intriguing: properly renormalized, the expected number of real zeros of $f_{n}$ does not converge as $n$ goes to infinity and admits in fact a whole continuum of subsequential limits. 
Zeros of trigonometric polynomials with strongly dependent Gaussian coefficients

Let us introduce the function $\ell^{\alpha}:(0, \pi) \rightarrow \mathbb{R}^{+}$defined by

$$
\ell^{\alpha}(x):=\frac{1}{4 \pi^{2}} \int_{[0,2 \pi]^{2}} \sqrt{1+\left|g_{n \alpha}^{\alpha}(s, u)\right|^{2}} d s d u
$$

where

$$
g_{x}^{\alpha}(s, u):=\frac{\sin (x) \sin \left(\frac{s-\alpha}{2}\right) \sin \left(\frac{s+\alpha}{2}\right)}{\sin ^{2}\left(\frac{u-x}{2}\right) \sin ^{2}\left(\frac{s+\alpha}{2}\right)+\sin ^{2}\left(\frac{u+x}{2}\right) \sin ^{2}\left(\frac{s-\alpha}{2}\right)} .
$$

In Section 3.1.1 below, we examine the properties of $\ell^{\alpha}$ and its pointwise limit as $\alpha$ goes to zero.

The main result of the paper is then the following one.

Theorem 1.1. For all $0<\beta<1$ and for all $n$ large enough such that $n \alpha \notin \pi \mathbb{Z}$, we have

$$
\left|\frac{\mathbb{E}\left[\mathcal{N}\left(f_{n},[0,2 \pi]\right)\right]}{n}-\ell^{a}(n \alpha \bmod \pi)\right|=O\left(\frac{1}{n^{\beta}(1-|\cos (n \alpha)|)^{2}}\right)+o(1) .
$$

The above theorem shows that if $n$ is sufficiently large but $n \alpha$ stays away enough from $\pi \mathbb{Z}$, then the expected number of real zeros on $f_{n}$ divided by $n$ is close to the value of the function $\ell^{\alpha}$ at the point $n \alpha \bmod \pi$. In particular, if $\alpha \in \pi \mathbb{Q}$, then the sequence $(n \alpha \bmod \pi)_{n \geq 1}$ takes values in a finite set $S$.

From the above Theorem 1.1, we can then deduce the following corollary.

Corollary 1.1. If $\alpha \in \pi \mathbb{Q}$, then for all $x \in S \backslash\{0\}$

$$
\lim _{n \rightarrow+\infty}\left|\frac{\mathbb{E}\left[\mathcal{N}\left(f_{n},[0,2 \pi]\right)\right]}{n}-\ell^{\alpha}(x)\right| \mathbb{1}_{n \alpha=x \bmod \pi}=0 .
$$

In particular $n^{-1} \mathbb{E}\left[\mathcal{N}\left(f_{n},[0,2 \pi]\right)\right]$ does not converge as $n$ goes to infinity.

Now if $\alpha \notin \pi \mathbb{Q}$, the sequence $(n \alpha \bmod \pi)_{n \geq 1}$ is dense in $[0, \pi]$ and from Theorem 1.1, one then deduces that $n^{-1} \mathbb{E}\left[\mathcal{N}\left(f_{n},[0,2 \pi]\right)\right]$ admits a whole continuum of possible limits.

Corollary 1.2. Let us fix $x \in(0, \pi)$ and consider a increasing subsequence $(\varphi(n))_{n \geq 1}$ such that $\varphi(n) \alpha$ converges to $x$ as $n$ goes to infinity. Then

$$
\lim _{n \rightarrow+\infty}\left|\frac{\mathbb{E}\left[\mathcal{N}\left(f_{\varphi(n)},[0,2 \pi]\right)\right]}{\varphi(n)}-\ell^{\alpha}(x)\right|=0 .
$$

Corollary 1.3. For all $\varepsilon>0$, for all $\ell \in(\sqrt{2}, 2]$, there exists $\alpha=\alpha(\ell) \geq 0$ small enough and infinitely many integers $n$ such that

$$
\left|\frac{\mathbb{E}\left[\mathcal{N}\left(f_{n},[0,2 \pi]\right)\right]}{n}-\ell\right| \leq \varepsilon
$$

where the Gaussian entries $\left(a_{k}\right)_{k \geq 1}$ and $\left(b_{k}\right)_{k \geq 1}$ of $f_{n}$ admit $\frac{\delta_{\alpha}+\delta_{-\alpha}}{2}$ as spectral measure.

Remark 1.1. For sake of clarity, we only deal here with a purely atomic spectral measure $\mu$ with two atoms $\pm \alpha$, but the method employed will work for any finite combination of atoms $\left( \pm \alpha_{i}\right)_{1 \leq i \leq N}$. The choice of a purely singular spectral measure could sound very particular but it actually dictates the fluctuating behavior of the expected number of zeros. Indeed, let us assume that the spectral measure $\mu$ can be written as the convex combination of a density measure and such a purely atomic measure, i.e.

$$
\mu=(1-\eta) \mu_{d}+\eta \frac{1}{N} \sum_{k=1}^{N} \frac{1}{2}\left(\delta_{\alpha_{k}}+\delta_{-\alpha_{k}}\right), \quad \eta \in[0,1), \alpha_{k} \geq 0,
$$


with $\mu_{d}$ admitting a density $\psi$ w.r.t. the Lebesgue measure on $[0,2 \pi]$ and satisfying the same assumptions as in [ADP19]. Then, combining the proof of the latter reference and the one of the present paper, one can show that

$$
\lim _{n \rightarrow+\infty} \frac{\mathbb{E}\left[\mathcal{N}\left(f_{n},[0,2 \pi]\right)\right]}{n}=\frac{2}{\sqrt{3}} .
$$

In other words, as soon as the spectral measure is not purely singular and have a density component, one recovers the universal asymptotics of the independent and weakly dependent case.

The rest of the paper is devoted to the proofs of the results stated above. In Section 2 , we give the proof of Proposition 1.1. Section 3 is devoted to the proof of the main Theorem 1.1 and its corollaries in the case where $n \alpha \notin \pi \mathbb{Z}$. In this case, the study of the number of zeros is split into two parts: in Section 3.1 we determine the number of zeros away from the atoms $\pm \alpha$ of the spectral measure $\mu$. Finally, the numbers of zeros in the neighborhood of the atoms is shown to be negligible in the last Section 3.2.

\section{Asymptotics in the case $n \alpha \in \pi \mathbb{Z}$}

In this Section, we give the proof of Proposition 1.1 describing the asymptotics of the number of real zeros of $f_{n}$ under the condition $n \alpha \in \pi \mathbb{Z}$. Let us first consider the very particular case where $\alpha=0$, i.e. the correlation function $\rho$ is constant equal to one.

Proposition 2.1. Suppose that $\alpha=0$, i.e. $\rho(k)=1$ for all $k \in \mathbb{N}$, then almost surely, for all $n \geq 1$ we have

$$
\mathcal{N}\left(f_{n},[0,2 \pi]\right)=2 n .
$$

Proof. Under the condition $\alpha=0$, the function $f_{n}$ has the simple form

$$
\begin{aligned}
f_{n}(t) & =\frac{1}{\sqrt{n}}\left(A \sum_{k=1}^{n} \cos (k t)+B \sum_{k=1}^{n} \sin (k t)\right) \\
& =\frac{1}{\sqrt{n}}\left(A \cos \left(\frac{n+1}{2} t\right)+B \sin \left(\frac{n+1}{2} t\right)\right) \frac{\sin (n t / 2)}{\sin (t / 2)} \text { a.s. }
\end{aligned}
$$

where $A, B$ are two independent standard Gaussian variables. Hence we count $n-1$ deterministic zeros corresponding to $\sin (n t / 2)=0$ and $n+1$ random zeros given by $t(\omega)=\frac{2 \pi}{n+1} U(\omega)+\frac{2 k \pi}{n+1}, k \in\{0, \ldots, n\}$, where $U=\pi / 2-\frac{1}{\pi} \arctan (-A / B)$ is uniform on $[0,1]$.

Let us now suppose that $\alpha=\frac{2 \pi p}{q}$ for positive and coprime integers $p$ and $q$, i.e. the correlation sequence $(\rho(k))_{k}$ is $q$-periodic. In this case, if $n=q r$ for some positive integer $r$, we have $n \alpha \in \mathbb{Z}$ and $f_{n}$ admits the following factorization

$$
f_{n}(t)=\frac{1}{\sqrt{n}} \sum_{k=1}^{q}\left(a_{k} \sum_{\ell=0}^{r-1} \cos ((\ell q+k) t)+b_{k} \sum_{\ell=0}^{r-1} \sin ((\ell q+k) t)\right)=\frac{1}{\sqrt{n}} \widetilde{f}_{n}(t) \times \frac{\sin \left(\frac{n t}{2}\right)}{\sin \left(\frac{q t}{2}\right)},
$$

where we have set

$$
\widetilde{f}_{n}(t):=\sum_{k=1}^{q} a_{k} \cos \left(k t+\frac{(n-q) t}{2}\right)+b_{k} \sin \left(k t+\frac{(n-q) t}{2}\right) .
$$

The above factorization of $f_{n}$ invites to distinguish deterministic and random zeros. We have $n-q$ deterministic zeros given by

$$
\sin \left(\frac{n t}{2}\right)=0 \text { and } \sin \left(\frac{q t}{2}\right) \neq 0 \Longleftrightarrow t \in\left\{\frac{2 k \pi}{n}, k \in\{0, \ldots, n-1\}, r \nmid k\right\} .
$$


Therefore the second statement in Proposition 1.1 follows from the following result which implies that, in the above framework, the expected number of real zeros of $\widetilde{f}_{n}$ is asymptotic to $n$.

Proposition 2.2. As $n$ tends to infinity, we have

$$
\liminf _{\substack{n \rightarrow+\infty \\ q \mid n}} \frac{1}{n} \mathbb{E}\left[\mathcal{N}\left(\widetilde{f}_{n},[0,2 \pi]\right)\right] \geq 1
$$

Proof. A direct computation shows that if $q \mid n$

$$
\mathbb{E}\left[\widetilde{f}_{n}(t)^{2}\right]=\frac{1}{2}\left[\frac{\sin ^{2}\left(\frac{q(\alpha+t)}{2}\right)}{\sin ^{2}\left(\frac{(\alpha+t)}{2}\right)}+\frac{\sin ^{2}\left(\frac{q(\alpha-t)}{2}\right)}{\sin ^{2}\left(\frac{(\alpha-t)}{2}\right)}\right] .
$$

Since $q \alpha \in \pi \mathbb{Z}$, we have thus for $t \in[0,2 \pi]$

$$
\mathbb{E}\left[\widetilde{f}_{n}(t)^{2}\right]=0 \Longrightarrow q t / 2 \in \pi \mathbb{Z} \Longrightarrow t \in S_{q}:=\left\{\frac{2 \pi k}{q}, 0 \leq k \leq q-1\right\} .
$$

For $\varepsilon>0$, set $S_{q}^{\varepsilon}:=\left\{t \in[0,2 \pi], \operatorname{dist}\left(t, S_{q}\right)>\varepsilon\right\}$. On $S_{q}^{\varepsilon}$, we have $\mathbb{E}\left[\widetilde{f}_{n}(t)^{2}\right]>0$ and applying Kac-Rice formula (see e.g. Theorem 3.2 p. 71 of [AW09]), we get

$$
\mathbb{E}\left[\mathcal{N}\left(\widetilde{f}_{n}, S_{q}^{\varepsilon}\right)\right]=\frac{1}{\pi} \int_{S_{q}^{\varepsilon}} \sqrt{\frac{\mathbb{E}\left[\tilde{f}_{n}^{\prime}(t)^{2}\right]}{\mathbb{E}\left[\widetilde{f}_{n}(t)^{2}\right]}-\left(\frac{\mathbb{E}\left[\tilde{f}_{n}(t) \tilde{f}_{n}^{\prime}(t)\right]}{\mathbb{E}\left[\widetilde{f}_{n}(t)^{2}\right]}\right)^{2}} d t .
$$

A straightforward computation shows that as $n$ goes to infinity, uniformly in $t \in S_{q}^{\varepsilon}$

$$
\mathbb{E}\left[\tilde{f}_{n}^{\prime}(t)^{2}\right]=\left(\frac{n-q}{2}\right)^{2} \mathbb{E}\left[\tilde{f}_{n}^{\prime}(t)^{2}\right]+o\left(n^{2}\right)
$$

Since $\mathbb{E}\left[\widetilde{f}_{n}(t)^{2}\right]$ does not depend on $n$, neither does $\mathbb{E}\left[\widetilde{f}_{n}(t) \widetilde{f}_{n}^{\prime}(t)\right]$ so that as $n$ goes to infinity, we have uniformly in $t \in S_{q}^{\varepsilon}$

$$
\sqrt{\frac{\mathbb{E}\left[\tilde{f}_{n}^{\prime}(t)^{2}\right]}{\mathbb{E}\left[\widetilde{f}_{n}(t)^{2}\right]}-\left(\frac{\mathbb{E}\left[\tilde{f}_{n}(t) \tilde{f}_{n}^{\prime}(t)\right]}{\mathbb{E}\left[\widetilde{f}_{n}(t)^{2}\right]}\right)^{2}}=\frac{n}{2}(1+o(1)) .
$$

Injecting this estimate in Equation (2.1), we deduce that as $n$ goes to infinity

$$
\frac{\mathbb{E}\left[\mathcal{N}\left(\tilde{f}_{n}, S_{q}^{\varepsilon}\right)\right]}{n}=\frac{\left|S_{q}^{\varepsilon}\right|}{2 \pi}(1+o(1))=1+O(\varepsilon)+o(1) .
$$

Letting $\varepsilon \rightarrow 0, \quad \liminf f_{n \rightarrow+\infty} \frac{\mathbb{E}\left[\mathcal{N}\left(\widetilde{f}_{n},[0,2 \pi]\right)\right.}{n} \geq \liminf _{n \rightarrow+\infty} \frac{\mathbb{E}\left[\mathcal{N}\left(\widetilde{f}_{n}, S_{q}^{\varepsilon}\right)\right]}{n}=1$.

\section{Asymptotics in the case $n \alpha \notin \pi \mathbb{Z}$}

We now consider the more intriguing case where $n \alpha \notin \pi \mathbb{Z}$. Following [ADP19], the variance and covariance of $\left(f_{n}(t), f_{n}^{\prime}(t)\right)$ can then be written as convolutions of the spectral measure $\mu$ with explicit trigonometric kernels, namely

$$
\mathbb{E}\left[f_{n}(t)^{2}\right]=K_{n} * \mu(t), \quad \mathbb{E}\left[f_{n}(t) f_{n}^{\prime}(t)\right]=\frac{1}{2} K_{n}^{\prime} * \mu(t), \quad \mathbb{E}\left[f_{n}^{\prime}(t)^{2}\right]=\frac{1}{\alpha_{n}} L_{n} * \mu(t),
$$


where $K_{n}(x):=\frac{1}{n}\left(\frac{\sin (n x / 2)}{\sin (x / 2)}\right)^{2}$ is the Fejer kernel, so that

$$
K_{n}^{\prime}(x):=\frac{2}{n}\left(\frac{\sin (n x / 2)}{\sin (x / 2)}\right)\left(\frac{n \cos (n x / 2)}{2 \sin (x / 2)}-\frac{\sin (n x / 2) \cos (x / 2)}{2 \sin (x / 2)^{2}}\right),
$$

the normalization constant $\alpha_{n}$ is given by $\alpha_{n}:=6 /(n+1)(2 n+1)$ and

$$
L_{n}(x):=\frac{\alpha_{n}}{n}\left|\sum_{k=0}^{n} k e^{i k x}\right|^{2}=\frac{\alpha_{n}}{n} \frac{(n+1)^{2}}{4 \sin (x / 2)^{2}}\left|1-\frac{\left(1-e^{i(n+1) x}\right) e^{-i n x}}{(n+1)\left(1-e^{i x}\right)}\right|^{2} .
$$

Lemma 3.1. For $0<\varepsilon \leq 1$, define $F_{\varepsilon}:=\{x \in[0,2 \pi],|\sin (x / 2)| \geq \varepsilon\}$. Then for all $n \geq 1$ such that $n \varepsilon>1$, we have the uniform estimates

$$
\sup _{x \in F_{\varepsilon}}\left|K_{n}^{\prime}(x)-\frac{\sin (n x / 2) \cos (n x / 2)}{\sin (x / 2)^{2}}\right|=O\left(\frac{1}{n \varepsilon^{3}}\right), \sup _{x \in F_{\varepsilon}}\left|L_{n}(x)-\frac{\alpha_{n} n}{4 \sin (x / 2)^{2}}\right|=O\left(\frac{1}{n^{2} \varepsilon^{3}}\right) .
$$

Proof. The estimate for $K_{n}^{\prime}$ is immediate. Since on $F_{\varepsilon}$,

$$
u:=\frac{\alpha_{n}}{n} \frac{(n+1)^{2}}{4 \sin (x / 2)^{2}} \leq \frac{\alpha_{n}}{n} \frac{(n+1)^{2}}{4 \varepsilon^{2}}, \quad z:=\frac{\left(1-e^{i(n+1) x}\right) e^{-i n x}}{(n+1)\left(1-e^{i x}\right)} \leq \frac{1}{(n+1)|\sin (x / 2)|} \leq \frac{1}{n \varepsilon},
$$

as soon as $n \varepsilon>1$, standard computations lead to

$$
\left|L_{n}(x)-u\right| \leq \frac{\alpha_{n}}{n} \frac{(n+1)^{2}}{4 \varepsilon^{2}} \times\left[\frac{3}{n \varepsilon}\right]=O\left(\frac{1}{n^{2} \varepsilon^{3}}\right) .
$$

Moreover, we have

$$
\left|\frac{\alpha_{n}}{n} \frac{(n+1)^{2}}{4 \sin (x / 2)^{2}}-\frac{\alpha_{n} n}{4 \sin (x / 2)^{2}}\right|=\frac{\alpha_{n}}{4 \sin (x / 2)^{2}}\left|\frac{(n+1)^{2}}{n}-n\right|=O\left(\frac{1}{n^{2} \varepsilon^{2}}\right)=O\left(\frac{1}{n^{2} \varepsilon^{3}}\right),
$$

hence the result.

In the case we consider here, the spectral measure $\mu$ is $\frac{1}{2}\left(\delta_{\alpha}+\delta_{-\alpha}\right)$ so that we have simply

$$
\begin{gathered}
\mathbb{E}\left[f_{n}(t)^{2}\right]=\frac{1}{2}\left(K_{n}(t-\alpha)+K_{n}(t+\alpha)\right), \mathbb{E}\left[f_{n}(t) f_{n}^{\prime}(t)\right]=\frac{1}{4}\left(K_{n}^{\prime}(t-\alpha)+K_{n}^{\prime}(t+\alpha)\right), \\
\text { and } \mathbb{E}\left[f_{n}^{\prime}(t)^{2}\right]=\frac{1}{2}\left(L_{n}^{\prime}(t-\alpha)+L_{n}^{\prime}(t+\alpha)\right) .
\end{gathered}
$$

The Fejér kernel being non negative, for $n \geq 1$, we have

$$
\mathbb{E}\left[f_{n}(t)^{2}\right]=0 \Rightarrow\left\{\begin{array}{r}
n t \in \pi \mathbb{Z} \\
n \alpha \in \pi \mathbb{Z} .
\end{array}\right.
$$

Under the assumption $n \alpha \notin \pi \mathbb{Z}$, the distribution of the Gaussian variable $f_{n}(t)$ is thus non-degenerated for all $t \in[0,2 \pi]$ and as above, we can use Kac-Rice formula (see e.g. [AW09]) to compute the expectation of $\mathcal{N}\left(f_{n},[0,2 \pi]\right)$, namely

$$
\mathbb{E}\left[\mathcal{N}\left(f_{n},[0,2 \pi]\right)\right]=\frac{1}{\pi} \int_{0}^{2 \pi} \sqrt{I_{n}(t)} d t
$$

where

$$
I_{n}(t):=\frac{1}{\alpha_{n}} \frac{L_{n}(t-\alpha)+L_{n}(t+\alpha)}{K_{n}(t-\alpha)+K_{n}(t+\alpha)}-\frac{1}{4}\left(\frac{K_{n}^{\prime}(t-\alpha)+K_{n}^{\prime}(t+\alpha)}{K_{n}(t-\alpha)+K_{n}(t+\alpha)}\right)^{2} .
$$

We split the computation of the integral into two parts, depending on the proximity between the integration variable $t$ and the atoms $\pm \alpha$ of the spectral measure $\mu$. 
Zeros of trigonometric polynomials with strongly dependent Gaussian coefficients

Remark 3.1. Alternatively, one can represent the processes $\left(a_{k}\right)_{k}$ and $\left(b_{k}\right)_{k}$ as

$$
a_{k}=\xi_{1} \cos (k \alpha)+\xi_{2} \sin (k \alpha), b_{k}=\xi_{3} \cos (k \alpha)+\xi_{4} \sin (k \alpha),
$$

where $\xi_{1}, \xi_{2}, \xi_{3}, \xi_{4}$ are independent standard Gaussian variables. In particular, the covariance function $r_{n}(t-s):=\mathbb{E}\left[f_{n}(t) f_{n}(s)\right]$ can be explicitly computed. The quantities involved in Kac-Rice formula thus correspond to $r_{n}(0), \partial_{t} \partial_{s} r_{n}(t-s)_{\mid t=s}$ and $\partial_{s} r_{n}(t-s)_{\mid t=s}$ and standard computations give the same expressions as given above.

\subsection{Away from the atoms}

Let us fix $\varepsilon>0$ and consider the set $J_{\varepsilon}:=\left\{t \in[0,2 \pi],\left|\sin \left(\frac{t-\alpha}{2}\right)\right|>\varepsilon,\left|\sin \left(\frac{t+a \alpha}{2}\right)\right|>\varepsilon\right\}$. Thanks to Lemma 3.1, we have then uniformly in $t \in J_{\varepsilon}$

$$
\frac{L_{n}(t-\alpha)+L_{n}(t+\alpha)}{K_{n}(t-\alpha)+K_{n}(t+\alpha)}=\frac{\frac{\alpha_{n} n^{2}}{4}\left(\frac{1}{\sin ^{2}\left(\frac{t-\alpha}{2}\right)}+\frac{1}{\sin ^{2}\left(\frac{t+\alpha}{2}\right)}\right)+O\left(\frac{1}{n \varepsilon^{3}}\right)}{\frac{\sin ^{2}\left(n \frac{t-\alpha}{2}\right)}{\sin ^{2}\left(\frac{t-\alpha}{2}\right)}+\frac{\sin ^{2}\left(n \frac{t+\alpha}{2}\right)}{\sin ^{2}\left(\frac{t+\alpha}{2}\right)}} .
$$

In the same manner, we have

$$
\frac{K_{n}^{\prime}(t-\alpha)+K_{n}^{\prime}(t+\alpha)}{K_{n}(t-\alpha)+K_{n}(t+\alpha)}=\frac{\frac{\sin \left(n \frac{t-\alpha}{2}\right) \cos \left(n \frac{t-\alpha}{2}\right)}{\sin ^{2}\left(\frac{t-\alpha}{2}\right)}+\frac{\sin \left(n \frac{t+\alpha}{2}\right) \cos \left(n \frac{t+\alpha}{2}\right)}{\sin ^{2}\left(\frac{t+\alpha}{2}\right)}+O\left(\frac{1}{n \varepsilon^{3}}\right)}{\frac{\sin ^{2}\left(n \frac{t-\alpha}{2}\right)}{n \sin ^{2}\left(\frac{t-\alpha}{2}\right)}+\frac{\sin ^{2}\left(n \frac{t+\alpha}{2}\right)}{n \sin ^{2}\left(\frac{t+\alpha}{2}\right)}} .
$$

Now remark that uniformly on $J_{\varepsilon}$ we have

$$
\begin{aligned}
\frac{1}{\frac{\sin ^{2}\left(n \frac{t-\alpha}{2}\right)}{\sin ^{2}\left(\frac{t-\alpha}{2}\right)}+\frac{\sin ^{2}\left(n \frac{t+\alpha}{2}\right)}{\sin ^{2}\left(\frac{t+\alpha}{2}\right)}} & \leq \frac{1}{\varepsilon^{2}\left(\sin ^{2}\left(n \frac{t-\alpha}{2}\right)+\sin ^{2}\left(n \frac{t+\alpha}{2}\right)\right)}=\frac{1}{\varepsilon^{2}(1-\cos (n t) \cos (n \alpha))} \\
& \leq \frac{1}{\varepsilon^{2}(1-|\cos (n \alpha)|) .}
\end{aligned}
$$

Therefore, uniformly on $J_{\varepsilon}$ we get

$$
I_{n}(t)=\frac{n^{2}}{4}\left(Q_{n}(t)+O\left(\frac{1}{n \varepsilon^{5}(1-|\cos (n \alpha)|)}\right)\right),
$$

where after standard calculations

$$
Q_{n}(t)=1+\left(\frac{\sin (n \alpha) \sin \left(\frac{t-\alpha}{2}\right) \sin \left(\frac{t+\alpha}{2}\right)}{\left(\sin ^{2}\left(n \frac{t-\alpha}{2}\right) \sin ^{2}\left(\frac{t+\alpha}{2}\right)+\sin ^{2}\left(n \frac{t+\alpha}{2}\right) \sin ^{2}\left(\frac{t-\alpha}{2}\right)\right)}\right)^{2} .
$$

In particular, we get

$$
\frac{2}{n} \int_{J_{\varepsilon}} \sqrt{I_{n}(t)} d t=\int_{J_{\varepsilon}} \sqrt{Q_{n}(t)} d t+O\left(\frac{1}{n \varepsilon^{5}(1-|\cos (n \alpha)|)}\right) .
$$

In order to make explicit the asymptotics of the right hand side of the last equation, let us now introduce an auxilary function and detail some of its properties.

\subsubsection{An auxilary function and its properties}

For $x \in \mathbb{R} \backslash \pi \mathbb{Z}$, let us introduce the function $g_{x}^{\alpha}$ defined on $[0,2 \pi]^{2} \backslash\{ \pm(\alpha, x)\}$ by

$$
g_{x}^{\alpha}(s, u):=\frac{\sin (x) \sin \left(\frac{s-\alpha}{2}\right) \sin \left(\frac{s+\alpha}{2}\right)}{\sin ^{2}\left(\frac{u-x}{2}\right) \sin ^{2}\left(\frac{s+\alpha}{2}\right)+\sin ^{2}\left(\frac{u+x}{2}\right) \sin ^{2}\left(\frac{s-\alpha}{2}\right)} .
$$


Zeros of trigonometric polynomials with strongly dependent Gaussian coefficients

Remark that $u \mapsto g_{x}^{\alpha}(s, u)$ is then $2 \pi$-periodic and that we have the identification

$$
Q_{n}(t)=1+\left|g_{n \alpha}^{\alpha}(t, n t)\right|^{2} .
$$

The function $(u, s) \mapsto g_{x}^{\alpha}(s, u)$ has singularities at $(s, u)= \pm(\alpha, x)$ but these sigularities are integrable in the following sense.

Lemma 3.2. Let $0<\alpha<\pi$ and $0<x<\pi$. For all $0 \leq \eta<1$, we have

$$
\int_{[0,2 \pi]^{2}}\left|g_{x}^{\alpha}(s, u)\right|^{1+\eta} d s d u<+\infty
$$

Proof. Let us fix some small $\delta>0$. Outside the two balls $B( \pm(\alpha, x), \delta)$ the function $(s, u) \mapsto g_{x}^{\alpha}(s, u)$ is uniformly bounded hence in $\mathbb{L}^{p}$ for all $p \geq 1$, so we only need to focus on the integrability on $B( \pm(\alpha, x), \delta)$. By symmetry, we can restrict ourselves to the ball centered at $(\alpha, x)$. If we set $C:=\min (|\sin (x)|,|\sin (\alpha)|)>0$, for $\delta$ small enough we have

$$
\left|g_{x}^{\alpha}(s, u)\right| \leq \frac{4}{C} \frac{|s-\alpha|}{|s-\alpha|^{2}+|u-x|^{2}},
$$

so that using polar coordinates $(s-\alpha, u-x)=(r \cos (\theta), r \sin (\theta))$ with $0 \leq r \leq \delta, 0 \leq \theta \leq 2 \pi$, we get

$$
\int_{B((\alpha, x), \delta)}\left|g_{x}^{\alpha}(s, u)\right|^{1+\eta} d s d u \leq \frac{8 \pi}{C} \int_{0}^{\delta} \frac{d r}{r^{\eta}}=O\left(\delta^{1-\eta}\right) .
$$

Lemma 3.3. On any compact set $K \subset(0, \pi)$, the function $\ell^{\alpha}: K \rightarrow \mathbb{R}^{+}$

$$
x \mapsto \ell^{\alpha}(x):=\frac{1}{4 \pi^{2}} \int_{[0,2 \pi]^{2}} \sqrt{1+\left|g_{x}^{\alpha}(s, u)\right|^{2}} d s d u
$$

is continuous.

Proof. Note that the regularity of $x \mapsto \ell^{\alpha}(x)$ is the same as the one of $x \mapsto \int_{[0,2 \pi]^{2}}\left|g_{x}^{\alpha}(s, u)\right| d s d u$. Fix $\varepsilon>0$, from the proof of Lemma 3.2 applied with $\eta=0$, there exists $\delta>0$ small enough such that, for all $x \in K$, if $E_{x}:=B((\alpha, x), \delta) \cup B(-(\alpha, x), \delta)$ then

$$
\int_{E_{x}}\left|g_{x}^{\alpha}(s, u)\right| d s d u \leq \varepsilon / 4
$$

Now, if $(s, u) \in E_{x}^{c} \cap E_{x^{\prime}}^{c}$ the function $x \mapsto\left|g_{x}^{\alpha}(s, u)\right|$ is uniformly bounded and analytic so that choosing $\delta>0$ small enough, for $\left|x-x^{\prime}\right|<\delta$ we have

$$
\left|\int_{E_{x}^{c} \cap E_{x^{\prime}}^{c}}\left(\left|g_{x}^{\alpha}(s, u)\right|-\left|g_{x^{\prime}}^{\alpha}(s, u)\right|\right) d s d u\right| \leq \varepsilon / 2 .
$$

The conclusion follows from this last estimate and triangular inequality.

The next lemma giving some properties of $g_{x}^{\alpha}$ which will be particularly useful in the sequel.

Lemma 3.4.

$$
\begin{gathered}
\sup _{\substack{s \in J_{\varepsilon} \\
u \in[0,2 \pi]}}\left|g_{x}^{\alpha}(s, u)\right|=O\left(\frac{1}{\varepsilon^{2}} \times \frac{1}{1-|\cos (x)|}\right), \\
\sup _{\substack{s, s^{\prime} \in J_{\varepsilon} \\
u \in[0,2 \pi]}}\left|g_{x}^{\alpha}(s, u)-g_{x}^{\alpha}\left(s^{\prime}, u\right)\right|=O\left(\frac{\left|s-s^{\prime}\right|}{\left.\varepsilon^{4}|1-| \cos (x) \mid\right)^{2}}\right) .
\end{gathered}
$$


Zeros of trigonometric polynomials with strongly dependent Gaussian coefficients

Proof. If $s \in J_{\varepsilon}$, we have uniformly in $u \in[0,2 \pi]$

$$
\left|g_{x}^{\alpha}(s, u)\right| \leq \frac{1}{\varepsilon^{2}\left[\sin ^{2}\left(\frac{u+x}{2}\right)+\sin ^{2}\left(\frac{u-x}{2}\right)\right]}=\frac{1}{\varepsilon^{2}(1-\cos (u) \cos (x))} \leq \frac{1}{\varepsilon^{2}} \times \frac{1}{1-|\cos (x)|} .
$$

Moreover, for $s, s^{\prime} \in J_{\varepsilon}$, setting $D(s):=\left(\sin ^{2}\left(\frac{u-x}{2}\right) \sin ^{2}\left(\frac{s+\alpha}{2}\right)+\sin ^{2}\left(\frac{u+x}{2}\right) \sin ^{2}\left(\frac{s-\alpha}{2}\right)\right)$

$$
\begin{aligned}
\left|g_{x}^{\alpha}(s, u)-g_{x}^{\alpha}\left(s^{\prime}, u\right)\right| & \leq \frac{\left|\sin \left(\frac{s-\alpha}{2}\right) \sin \left(\frac{s+\alpha}{2}\right)-\sin \left(\frac{s^{\prime}-\alpha}{2}\right) \sin \left(\frac{s^{\prime}+\alpha}{2}\right)\right|}{D(s)}+\frac{\left|D(s)-D\left(s^{\prime}\right)\right|}{\left|D(s) D\left(s^{\prime}\right)\right|} \\
& =O\left(\frac{\left|s-s^{\prime}\right|}{\varepsilon^{2}(1-|\cos (x)|)}\right)+O\left(\frac{\left|s-s^{\prime}\right|}{\varepsilon^{4}(1-|\cos (x)|)^{2}}\right) .
\end{aligned}
$$

For $x \in(0, \pi)$, set

$$
\ell^{0}(x):=\frac{1}{2 \pi} \int_{0}^{2 \pi} \sqrt{1+g_{x}^{0}(u)^{2}} d u, \quad \text { where } \quad g_{x}^{0}(u):=\frac{\sin (x)}{1-\cos (u) \cos (x)} .
$$

We show now that the function $\ell^{0}$ appears naturally as the pointwise limit of $\ell^{\alpha}$ given in Section 1.2 when $\alpha \in(0, \pi)$ goes to zero.

Lemma 3.5. For all $x \in(0, \pi)$, we have $\lim _{\alpha \rightarrow 0} \ell^{\alpha}(x)=\ell^{0}(x)$.

Proof. Let $\epsilon>0$ and let $\alpha \in\left(0, \frac{\epsilon}{2}\right)$ be small enough. We can write

$$
\ell^{\alpha}(x)=\frac{1}{4 \pi^{2}}\left[\int_{|s|>\epsilon} \int_{-\pi}^{\pi} \sqrt{1+g_{x}^{\alpha}(s, u)^{2}} d s d u+\int_{|s| \leq \epsilon} \int_{-\pi}^{\pi} \sqrt{1+g_{x}^{\alpha}(s, u)^{2}} d s d u\right] .
$$

For $|s|>\epsilon$, there exists a constant $C>0$ such that $\left|\sin \left(\frac{s \pm \alpha}{2}\right)\right| \geq C \epsilon$. By dominated convergence (using Lemma 3.4 for the upper bound), we first obtain

$$
\lim _{\alpha \rightarrow 0} \int_{|s|>\epsilon} \int_{-\pi}^{\pi} \sqrt{1+g_{x}^{\alpha}(s, u)} d s d u=2(\pi-\epsilon) \int_{-\pi}^{\pi} \sqrt{1+\frac{\sin ^{2}(x)}{(1-\cos (u) \cos (x))^{2}}} d s .
$$

Let us now show that the second term in Equation (3.6) converges to zero as $\alpha$ goes to zero. By symmetry, we can restrict ourselves to the case $s \in[0, \epsilon]$. This way, $s \pm \alpha$ is close to zero. Thus, there exists $C>0$ such that

$$
\left|g_{x}^{\alpha}(s, u)\right| \leq C \frac{|\sin (x)||(s-\alpha)(s+\alpha)|}{\sin ^{2}\left(\frac{u-x}{2}\right)(s+\alpha)^{2}+\sin ^{2}\left(\frac{u+x}{2}\right)(s-\alpha)^{2}} .
$$

Set $\delta>0$ small enough such that for all $u \in[x-\delta, x+\delta]$, we have $\left|\sin \left(\frac{u-x}{2}\right)\right| \geq C_{\delta}|u-x|$ and $\left|\sin \left(\frac{u+x}{2}\right)\right| \geq C_{\delta} \sin (x)$. Using the fact that $s+\alpha \geq \alpha$, we get that for some the constant $C$ which may change from line to line

$$
\begin{aligned}
\int_{0}^{\epsilon} \int_{x-\delta}^{x+\delta}\left|g_{x}^{a}(s, u)\right| d u d s & \leq C \int_{0}^{\epsilon} \int_{x-\delta}^{x+\delta} \frac{\left|s^{2}-\alpha^{2}\right|}{(s-\alpha)^{2}+\alpha^{2}(u-x)^{2}} d u d s \\
& \leq C \int_{0}^{\epsilon} \frac{|s+\alpha|}{\alpha} \arctan \left(\frac{\delta \alpha}{|s-\alpha|}\right) d s \\
& \leq \int_{0}^{\epsilon} \frac{|s-\alpha|}{\alpha} \arctan \left(\frac{\delta \alpha}{|s-\alpha|}\right) d s+2 \underbrace{\int_{0}^{\epsilon} \arctan \left(\frac{\delta \alpha}{|s-\alpha|}\right) d s}_{\leq C \epsilon} \\
& \leq \epsilon \times \frac{\alpha}{\epsilon} \int_{-\frac{\epsilon}{\alpha}}^{\frac{\epsilon}{\alpha}}|v| \arctan \left(\frac{\delta}{|v|}\right) d v+C \epsilon \leq C \epsilon .
\end{aligned}
$$


thanks to the change of variable $v=\frac{s-\alpha}{\alpha}$ and the fact that $x \mapsto x \arctan \left(\frac{1}{x}\right)$ is bounded on $\mathbb{R}$.

The same method naturally works in the neighborhood of $-x$. Otherwise, if we denote by $E_{\delta}$ the set $([x-\delta, x+\delta] \cup[-x-\delta,-x+\delta])^{c}$, there exists a constant $C_{x, \delta}$ such that for all $u$ in $E_{\delta}$, we have $\left|\sin \left(\frac{u \pm x}{2}\right)\right| \geq C_{x, \delta}$. Thus, for some constant which may again change from line to line, we get

$$
\begin{aligned}
\int_{0}^{\epsilon} \int_{E_{\delta}}\left|g_{x}^{\alpha}(s, u)\right| d s d u & \leq C \int_{0}^{\epsilon} \frac{\left|s^{2}-\alpha^{2}\right|}{(s-\alpha)^{2}+(s+\alpha)^{2}} d s \\
& \leq C \underbrace{\int_{0}^{\epsilon} \frac{\left|s^{2}+\alpha^{2}\right|}{s^{2}+\alpha^{2}} d s}_{=\epsilon}+2 \alpha^{2} \int_{0}^{\epsilon} \frac{d s}{s^{2}+\alpha^{2}} \leq C(\epsilon+\alpha) \leq C \epsilon,
\end{aligned}
$$

hence the result.

Let us conclude this section with some properties of the limit function $\ell^{0}(x)$.

Lemma 3.6. The function $x \mapsto \ell^{0}(x)$ is analytic on $(0, \pi)$ and admits $x=\frac{\pi}{2}$ as a symmetry axis. Moreover, $[\sqrt{2}, 2) \subseteq \ell^{0}[(0, \pi)]$.

Proof. Analyticity follows form standard dominated convergence. Using the change of variable $v=u+\pi$ and $2 \pi$-periodicity of the integrand, we get that for all $z \in$ $\left[0, \frac{\pi}{2}\right), \ell^{0}\left(z+\frac{\pi}{2}\right)=\ell^{0}\left(\frac{\pi}{2}-z\right)$. Therefore $x=\frac{\pi}{2}$ is a symmetry axis.

The inequality $\ell(x) \leq 2$ results from the fact that

$$
\frac{1}{2 \pi} \int_{0}^{2 \pi} \frac{\sin (x)}{1-\cos (u) \cos (x)} d u=1 .
$$

In fact, the upper value 2 is obtained as the limit on the boundaries.

Set $\delta>0$ and let $x$ be small enough. We can indeed write

$$
\ell^{0}(x)=\frac{1}{2 \pi} \int_{[-\pi, \pi] \backslash[-\delta, \delta]} \sqrt{1+g_{x}^{0}(u)^{2}} d u+\frac{1}{2 \pi} \int_{-\delta}^{\delta} \sqrt{1+g_{x}^{0}(u)^{2}} d u .
$$

By dominated convergence (using the upper bound for $g_{x}^{0}$ as in Lemma 3.4),

$$
\lim _{x \rightarrow 0} \frac{1}{2 \pi} \int_{[-\pi, \pi] \backslash[-\delta, \delta]} \sqrt{1+\frac{\sin ^{2}(x)}{(1-\cos (x) \cos (u))^{2}}} d u=1 .
$$

On the other hand, we can assume that $\int_{-\delta}^{\delta} \sqrt{1+g_{x}^{2}(u)} d u \geq \int_{-\sqrt{x}}^{\sqrt{x}} \sqrt{1+g_{x}^{2}(u)} d u$ for $x$ small enough.

Then, we get

$$
\begin{aligned}
\frac{1}{2 \pi} \int_{-\sqrt{x}}^{\sqrt{x}} \sqrt{1+g_{x}^{2}(u)} d u & =\frac{1}{\pi} \int_{0}^{\sqrt{x}} \sqrt{1+g_{x}^{2}(u)} d u \geq \frac{\sin (x)}{\pi} \int_{0}^{\sqrt{x}} \frac{\sin (x)}{1-\cos (u) \cos (x)} d u \\
& \geq \frac{2}{\pi} \sin (x) \int_{0}^{\sqrt{x}} \frac{d u}{u^{2}+x^{2}}=\frac{2}{\pi} \times \frac{\sin (x)}{x} \times \arctan \left(\frac{\sqrt{x}}{x}\right)
\end{aligned}
$$

since $1-\cos (u) \cos (x) \leq \frac{u^{2}+x^{2}}{2}$. Hence we get

$$
\lim _{x \rightarrow 0} \frac{1}{2 \pi} \int_{[-\delta, \delta]} \sqrt{1+g_{x}^{2}(u)} d u \geq 1,
$$

Finally, combining the estimates (3.7) and (3.8), we obtain $\lim _{x \rightarrow 0} \ell^{0}(x)=2$. The analogue limit as $x$ tends to $\pi$ is deduced by symmetry. Since $\ell^{0}(\pi / 2)=\sqrt{2}$ and $\ell^{0}$ is continuous, the intermediate value theorem yields that $[\sqrt{2}, 2) \subset \ell^{0}[(0, \pi)]$. 
Zeros of trigonometric polynomials with strongly dependent Gaussian coefficients

\subsubsection{From Riemann sum to integral}

We can now establish the asymptotics of Equation (3.2) as $n$ goes to infinity. As a first step, the integral of interest admits the following lower and upper bounds.

Lemma 3.7. If $n \varepsilon>>1$, then as $n$ goes to infinity, we have

$$
\int_{J_{\varepsilon}} \sqrt{Q_{n}(t)} d t \geq \frac{1}{2 \pi} \int_{[0,2 \pi]^{2}} \sqrt{1+\left|g_{n \alpha}^{\alpha}(s, u)\right|^{2}} \mathbb{1}_{s \in J_{2 \varepsilon}} d s d u+O\left(\frac{1}{n \varepsilon^{2}(1-|\cos (n \alpha)|)}\right),
$$

and

$$
\int_{J_{\varepsilon}} \sqrt{Q_{n}(t)} d t \leq \frac{1}{2 \pi} \int_{[0,2 \pi]^{2}} \sqrt{1+\left|g_{n \alpha}^{\alpha}(s, u)\right|^{2}} \mathbb{1}_{s \in J_{\varepsilon / 2}} d s d u+O\left(\frac{1}{n \varepsilon^{2}(1-|\cos (n \alpha)|)}\right) .
$$

Proof. We give the proof of the upper bound, the lower bound can be treated in the exact same way. To simplify the expressions, let us set $E_{n}^{k}:=\left[\frac{2 \pi k}{n}, \frac{2 \pi(k+1)}{n}\right]$ for $0 \leq k \leq n-1$. We can then decompose the integral on $J_{\varepsilon}$ as

$$
\int_{J_{\varepsilon}} \sqrt{Q_{n}(t)} d t=\sum_{k=0}^{n-1} \int_{J_{\varepsilon} \cap E_{n}^{k}} \sqrt{Q_{n}(t)} d t=\frac{1}{n} \sum_{k=0}^{n-1} \int_{0}^{2 \pi} \sqrt{Q_{n}\left(\frac{2 \pi k}{n}+\frac{u}{n}\right)} \mathbb{1}_{\frac{2 \pi k+u}{n} \in J_{\varepsilon}} d u .
$$

Now remark that if $n \varepsilon>>1$, then for $n$ large enough, if $\frac{2 \pi k+u}{n} \in J_{\varepsilon}$ we have in fact $E_{n}^{k} \subset J_{\varepsilon / 2}$. Therefore

$$
\begin{aligned}
\int_{J_{\varepsilon}} \sqrt{Q_{n}(t)} d t & \leq \frac{1}{n} \sum_{k=0}^{n-1} \int_{0}^{2 \pi} \sqrt{Q_{n}\left(\frac{2 \pi k}{n}+\frac{u}{n}\right)} \mathbb{1}_{E_{n}^{k} \subset J_{\varepsilon / 2}} d u \\
& \leq \frac{1}{n} \sum_{k=0}^{n-1} \int_{0}^{2 \pi} \sqrt{1+g_{n \alpha}^{\alpha}\left(\frac{2 \pi k}{n}+\frac{u}{n}, u\right)} \mathbb{1}_{E_{n}^{k} \subset J_{\varepsilon / 2}} d u
\end{aligned}
$$

thank to (3.4) and the $2 \pi$-periodicity of $u \mapsto g_{n \alpha}^{\alpha}(s, u)$.

Using the estimate (3.5) of Lemma 3.4, one then deduces that

$$
\int_{J_{\varepsilon}} \sqrt{Q_{n}(t)} d t \leq \frac{1}{n} \sum_{k=0}^{n-1} \int_{0}^{2 \pi} \sqrt{1+g_{n \alpha}^{\alpha}\left(\frac{2 \pi k}{n}, u\right)} \mathbb{1}_{E_{n}^{k} \subset J_{\varepsilon / 2}} d u+O\left(\frac{1}{\left.n \varepsilon^{4}|1-| \cos (n \alpha) \mid\right)^{2}}\right) .
$$

Using again Equation (3.5) of Lemma 3.4, for all $0 \leq k \leq n-1$ such that $E_{n}^{k} \subset J_{\varepsilon / 2}$, we have uniformly in $u$

$$
\left|\sqrt{1+g_{n \alpha}^{\alpha}\left(\frac{2 \pi k}{n}, u\right)}-\frac{n}{2 \pi} \int_{E_{n}^{k}} \sqrt{1+g_{n \alpha}^{\alpha}(s, u)} d s\right|=O\left(\frac{1}{\left.n \varepsilon^{4}|1-| \cos (n \alpha) \mid\right)^{2}}\right) .
$$

Integrating in $u$, we thus get that for all $k$ such that $E_{n}^{k} \subset J_{\varepsilon / 2}$,

$\int_{0}^{2 \pi} \sqrt{1+g_{n \alpha}^{\alpha}\left(\frac{2 \pi k}{n}, u\right)} d u \leq \frac{n}{2 \pi} \int_{0}^{2 \pi} \int_{E_{n}^{k}} \sqrt{1+g_{n \alpha}^{\alpha}(s, u)} d s d u+O\left(\frac{1}{\left.n \varepsilon^{4}|1-| \cos (n \alpha) \mid\right)^{2}}\right)$,

and in particular

$$
\begin{aligned}
\int_{0}^{2 \pi} \sqrt{1+g_{n \alpha}^{\alpha}\left(\frac{2 \pi k}{n}, u\right)} d u \times \mathbb{1}_{E_{n}^{k} \subset J_{\varepsilon / 2}} & \leq \frac{n}{2 \pi} \int_{0}^{2 \pi} \int_{E_{n}^{k}} \sqrt{1+g_{n \alpha}^{\alpha}(s, u)} \mathbb{1}_{s \in J_{\varepsilon / 2}} d s d u \\
& +O\left(\frac{1}{\left.n \varepsilon^{4}|1-| \cos (n \alpha) \mid\right)^{2}}\right) .
\end{aligned}
$$


Injecting this last estimate in Equation (3.9) and making the sum over $0 \leq k \leq n-1$, we get

$$
\int_{J_{\varepsilon}} \sqrt{Q_{n}(t)} d t \leq \frac{1}{2 \pi} \int_{[0,2 \pi]^{2}} \sqrt{1+g_{n \alpha}^{\alpha}(s, u)} \mathbb{1}_{s \in J_{\varepsilon / 2}} d s d u+O\left(\frac{1}{\left.n \varepsilon^{4}|1-| \cos (n \alpha) \mid\right)^{2}}\right) .
$$

Lemma 3.8. Uniformly in $n$, and for all $0<\eta<1$, we have

$$
\left|\int_{[0,2 \pi]^{2}} \sqrt{1+\left|g_{n \alpha}^{\alpha}(s, u)\right|^{2}} \mathbb{1}_{s \in J_{\varepsilon}} d s d u-\int_{[0,2 \pi]^{2}} \sqrt{1+\left|g_{n \alpha}^{\alpha}(s, u)\right|^{2}} d s d u\right|=O\left(\varepsilon^{\frac{\eta}{1+\eta}}\right) .
$$

Proof. It results from applying Hölder inequality with $p=1+\eta$ and $q=1+1 / \eta$ and using Lemma 3.2.

Combining the estimate (3.2) and Lemmas 3.7 and 3.8, we conclude that for all $\varepsilon>0$ and $n$ large enough such that $n \varepsilon>>1$ then

$$
\left|\frac{4 \pi}{n} \int_{J_{\varepsilon}} \sqrt{I_{n}(t)} d t-\int_{[0,2 \pi]^{2}} \sqrt{1+g_{n \alpha}^{\alpha}(s, u)^{2}} d s d u\right|=O\left(\varepsilon^{\frac{\eta}{1+\eta}}\right)+O\left(\frac{1}{\left.n \varepsilon^{5}|1-| \cos (n \alpha) \mid\right)^{2}}\right) .
$$

\subsection{Near the atoms and conclusion}

We are left to estimate the number of real zeros of $f_{n}$ in the neighborhood of the atoms $\pm \alpha$ of the spectral measure $\mu$. If $\varepsilon=\varepsilon_{n}$ is of the form $\varepsilon_{n}=n^{-\beta}$ with $0<\beta<1 / 2$, Proposition 3.3.1 of [Pir19a] indeed show that

$$
\frac{\mathbb{E}\left[\mathcal{N}\left(f_{n}, J_{\varepsilon_{n}}^{c}\right)\right]}{n}=O\left(\varepsilon_{n}\right) .
$$

Therefore, we can conclude that, as soon as $\varepsilon_{n}$ is chosen of the form $n^{-\beta}$ for $0<\beta<1 / 5$, we have

$$
\left|\frac{\mathbb{E}\left[\mathcal{N}\left(f_{n},[0,2 \pi]\right)\right]}{n}-\ell^{\alpha}(n \alpha \bmod \pi)\right|=O\left(\varepsilon_{n}^{\frac{\eta}{1+\eta}}\right)+O\left(\frac{1}{\left.n \varepsilon_{n}^{5}|1-| \cos (n \alpha) \mid\right)^{2}}\right),
$$

which finishes the proof of Theorem 1.1. Then Corollary 1.1 follows because uniformly in $x \in S \backslash\{0\}$, if $n \alpha \bmod \pi=x$, then $1-|\cos (n \alpha)|=1-|\cos (x)|$ is bounded away from zero. In the last case where $\alpha \notin \pi \mathbb{Q}$, Corollary 1.2 follows from Theorem 1.1 and the regularity of $\ell^{\alpha}$ established in Lemma 3.3.

From Lemmas 3.6, 3.5 and the estimate (3.11) as $\alpha \rightarrow 0$ and $n \alpha \bmod \pi \rightarrow 0$, remark that we get the same limit (1.1) as in Proposition 1.1. In the same manner, Corollary 1.3 follows from Corollary 1.2, Lemmas 3.5 and 3.6 for $\ell \in(\sqrt{2}, 2)$ and from Proposition 1.1 for $\ell=2$.

\section{References}

[ADL] J.M Azaïs, F. Dalmao, J.R Leon, I. Nourdin and G. Poly, Local universality of the number of zeros of random trigonometric polynomials with continuous coefficients, arXiv:1512.05583.

[ADP19] J. Angst, F. Dalmao and G. Poly, On the real zeros of random trigonometric polynomials with dependent coefficients, Proc. Amer. Math. Soc. 147 (2019), no. 1, 205-214. MR-3876743

[AP15] J. Angst and G. Poly, Universality of the mean number of real zeros of random trigonometric polynomials under a weak cramer condition, arXiv:1511.08750, 2015 MR-0336811 
Zeros of trigonometric polynomials with strongly dependent Gaussian coefficients

[AW09] J-M. Azaïs and M. Wschebor, Level sets and extrema of random processes and fields, Chap. 3 p71. MR-2478201

[DNV18] Y. Do, O. Nguyen and V. Vu, Roots of random polynomials with coefficients of polynomial growth, Ann. Probab., Vol 46, Number 5, 2407-2494, 2018. MR-3846831

[Dun66] J.E.A. Dunnage, The number of real zeros of a random trigonometric polynomial, Proc. London Math. Soc. (3), 16:53-84, 1966 MR-0192532

[Far86] K. Farahmand, On the average number of real roots of a random algebraic equation, Ann. Prob., 14(2):702-709, 1986. MR-0832032

[FL12] K. Farahmand and T. Li, Real zeros of three different cases of polynomials with random coefficients, Rocky Mountain J. Math. 42 (2012), 1875-1892. MR-3028766

[Fla17] H. Flasche, Expected number of real roots of random trigonometric polynomials, Stochastic Processes and their Applications, pages -, 2017 MR-3718101

[IKM16] A. Iksanov, Z. Kabluchko and A. Marynych, Local universality for real roots of random trigonometric polynomials, Electron. J. Probab., Vol 21, paper no 63,19 pp, 2016 MR-3563891

[IM68] I.A. Ibragimov and N.B. Maslova, On the expected number of real zeros of random polynomials i. coefficients with zero means, Theory of Probability and Its Applications, 16(2):228-248, 1971. MR-0286157

[Mat10] J. Matayoshi, The real zeros of a random algebraic polynomial with dependent coefficients, Rocky Mountain J. Math. 42, No 3, pp. 1015-1034 MR-2966483

[Muk18] S. Mukeru, Average number of real zeros of random algebraic polynomials defined by the increments of fractional Brownian motion, J. Th. Prob., p.1-23, 2018 MR-3979678

[NNV15] H. Nguyen, O. Nguyen and V.Vu, On the number of real roots of random polynomials, Com. in Cont. Math. Vol 18, 1550052, 2015. MR-3493213

[Kac43] M. Kac. On the average number of real roots of a random algebraic equation, Bull. Amer. Math. Soc. 49:314-320, 1943 MR-0007812

[Pir19a] A. Pirhadi, Real zeros of random trigonometric polynomials with pairwise equal blocks of coefficients, arXiv:1905.13349, 2019

[Pir19b] A. Pirhadi, Real zeros of random cosine polynomials with palindromic blocks of coefficients, arXiv:1908.08154, to appear in Rocky Mountain J. Math., 2020

[RS84] N. Renganathan and M. Sambandham, On the average number of real zeros of a random trigonometric polynomial with dependent coefficients, Indian Journal of Pure and Applied Mathematrics, 15(9):951-956, 1984 MR-0761283

[Sam78] M. Sambandham, On the number of real zeros of a random trigonometric polynomial, Trans. Amer. Math. Soc., 238:57-70, 1978 MR-0461648 


\section{Electronic Journal of Probability Electronic Communications in Probability}

\section{Advantages of publishing in EJP-ECP}

- Very high standards

- Free for authors, free for readers

- Quick publication (no backlog)

- Secure publication $\left(\mathrm{LOCKSS}^{1}\right)$

- Easy interface (EJMS²)

\section{Economical model of EJP-ECP}

- Non profit, sponsored by $\mathrm{IMS}^{3}, \mathrm{BS}^{4}$, ProjectEuclid ${ }^{5}$

- Purely electronic

\section{Help keep the journal free and vigorous}

- Donate to the IMS open access fund ${ }^{6}$ (click here to donate!)

- Submit your best articles to EJP-ECP

- Choose EJP-ECP over for-profit journals

\footnotetext{
${ }^{1}$ LOCKSS: Lots of Copies Keep Stuff Safe http://www. lockss.org/

${ }^{2}$ EJMS: Electronic Journal Management System http://www.vtex.lt/en/ejms.html

${ }^{3}$ IMS: Institute of Mathematical Statistics http://www.imstat.org/

${ }^{4}$ BS: Bernoulli Society http://www. bernoulli-society.org/

${ }^{5}$ Project Euclid: https://projecteuclid.org/

${ }^{6}$ IMS Open Access Fund: http://www.imstat.org/publications/open.htm
} 